\title{
Clinical Characteristics and Thoracic factors in patients with Idiopathic and Syndromic Scoliosis Associated with Pectus Excavatum
}

\author{
Ryoji Tauchi ${ }^{1)}$, Yoshitaka Suzuki ${ }^{2)}$, Taichi Tsuji ${ }^{1}$, Tetsuya Ohara ${ }^{1)}$, Toshiki Saito ${ }^{1}$, \\ Ayato Nohara $^{3)}$, Kazuaki Morishita ${ }^{1)}$, Ippei Yamauchi ${ }^{1)}$ and Noriaki Kawakami ${ }^{1)}$ \\ 1) Department of Orthopedic Surgery, Meijo Hospital, Aichi, Japan \\ 2) Department of Orthopedic Surgery, Nagoya Daini Red Cross Hospital, Aichi, Japan \\ 3) Department of Orthopedic Surgery, JCHO Tokyo Shinjuku Medical Center, Tokyo, Japan
}

\begin{abstract}
:
Introduction: The purpose of this study is to demonstrate the clinical characteristics and thoracic factors such as sternal tilt angle and Haller index in patients with idiopathic or syndromic scoliosis associated with pectus excavatum.

Methods: We performed a retrospective review on a cohort of 70 patients (37 males and 33 females) diagnosed with idiopathic and syndromic scoliosis associated with pectus excavatum between 1985 and 2014. We investigated age, location and Cobb angle of the main curve, and thoracic factors including sternal deviation and tilting angle and Haller index using radiographs and computed tomography of the chest.

Results: Patients' mean age at the first visit to our hospital was 10.3 years (1-18 years old). There were 41 patients with idiopathic scoliosis and 29 with syndromic scoliosis. Main curve locations were thoracic in 52 patients, thoracolumbar in 10, and lumbar in 8. The mean Cobb angle of the main curve was 45.0 degrees (11-109 degrees). The sternum was displaced on the left side in $72 \%$ of patients, central in $23 \%$, and right in 5\%. Mean sternal tilt angle was 12.4 degrees (2.3-34 degrees), and mean Haller index score was 4.9 (2.9-9.2). There was no significant correlation between Cobb angle and sternal tilt angle/Haller index. However, a significant difference was found between sternal tilt angle and Haller index.

Conclusion: Most patients with both scoliosis and pectus excavatum have left side deviated sternum and a higher Haller index score; therefore this can negatively impact cardiac function. Prone positioning and the corrective force applied during scoliosis surgery as well as thoracic compression during cast or brace treatment may have a negative effect on cardiac function in these patients.
\end{abstract}

Keywords:

scoliosis, pectus excavatum, sternum, Haller index

Spine Surg Relat Res 2018; 2(1): 37-41 dx.doi.org/10.22603/ssrr.2017-0027

\section{Introduction}

Pectus excavatum is the most common morphological chest wall abnormality with a reported incidence of 0.1 $0.3 \%^{1,2}$. The pathogenesis of pectus excavatum is unknown, but it appears to be polygenetic following autosomal dominant, autosomal recessive, X-linked, or sporadic patterns of inheritance ${ }^{3,4)}$. Several studies on pectus excavatum patients evaluated the coexistence of scoliosis and concluded that the rate of scoliosis in patients with pectus excavatum was higher than the rate in the general population ${ }^{5-9)}$. Gurnett CA et al. found that adolescent idiopathic scoliosis and pectus excavatum may have a similar genetic etiology, and the prevalence of scoliosis among pectus excavatum patients is higher than the prevalence within the general population ${ }^{10}$. Furthermore, pectus excavatum is frequently associated with heritable disorders of the connective tissue, such as Marfan syndrome in which almost half the patients have pectus excavatum and a large number have scoliosis ${ }^{11-15)}$. However, to our knowledge, although a few studies have reported on the clinical characteristics of patients having both scoliosis and pectus excavatum, they do not give a detailed discussion and

Corresponding author: Noriaki Kawakami, nupriver@gmail.com

Received: April 6, 2017, Accepted: June 23, 2017

Copyright (C) 2018 The Japanese Society for Spine Surgery and Related Research 
the degree of scoliosis is not severe ${ }^{6,8,9,16,17)}$. The purpose of this study is to demonstrate clinical features and thoracic factors, such as sternal tilt angle and Haller index, in patients that have idiopathic or syndromic scoliosis associated with pectus excavatum.

\section{Methods}

\section{Patient Selection and Clinical Investigation}

From April 1985 to February 2014, 113 patients with both scoliosis and pectus excavatum were admitted to our hospital. We excluded adult scoliosis (age more than 20 years), congenital scoliosis, neuromuscular scoliosis, neurofibromatosis type 1 , scoliosis of less than 10 degrees, and patients with incomplete clinical data or images. A total of 70 patients (37 male and 33 female) that had idiopathic or syndromic scoliosis associated with pectus excavatum were evaluated. We investigated age, location and Cobb angle of the main curve, thoracic factors including the deviation and tilting angle of the sternum, and the Haller index using radiographs and computed tomography of the chest. We also evaluated whether patients received surgical correction of their pectus excavatum.

\section{Radiologic Evaluation}

CT scans were used to determine sternal deviation (left, center, right), sternal tilt (left or right inclination and angle), and Haller index. We evaluated sternal deviation and tilt in 53 patients, but we were only able to estimate sternal tilt angle and Haller index in 29 patients because some cases did not have an image of the entire chest wall and others did not have CT images taken prior to corrective surgery for the pectus excavatum. We defined sternal tilt as the angle generated by the sternum and the axis of the body at the most collapsed plane of the sternum in axial CT scans (Fig. 1). Position of the sternum was defined as right, center, or left based on the position of the center of the sternum relative to

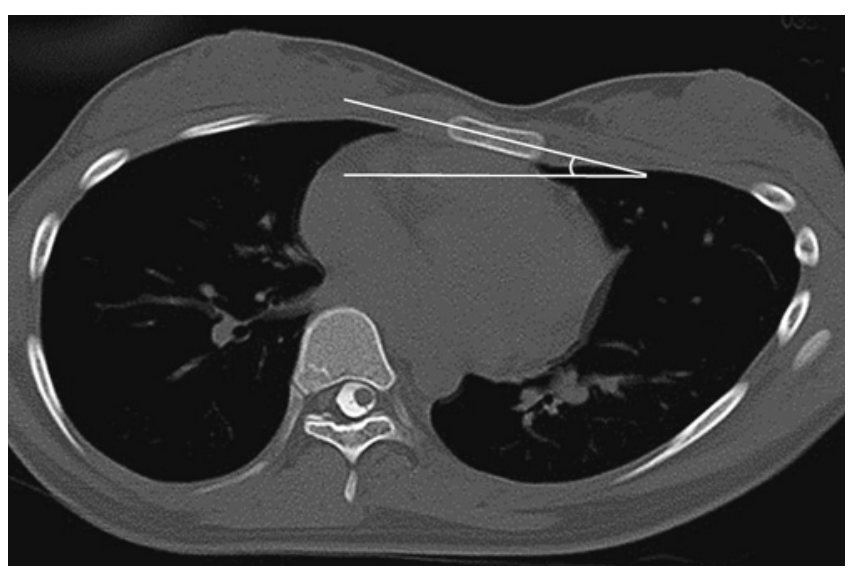

Figure 1. Sternal tilt angle. Axial CT scan showing the angle generated by the sternum and the axis of the body at the most depressed plane of the sternum. the vertebral body on axial CT scan.

We calculated the Haller index as the inner transverse thoracic diameter divided by the anteroposterior distance between the anterior thoracic wall and the spine at the narrowest point (Fig. 2). In normal individuals, the average Haller index is 2.5 , whereas with a significant pectus deformity it is 3.25 or greater ${ }^{18)}$. In patients with scoliosis and pectus excavatum, since the sternum tends to be deviated unilaterally, we modified the method for calculating the Haller index (Modified Haller index; Fig. 2). Two senior spine surgeons (R.T., Y.S.) measured each parameter twice, and the average score was used for analysis. The intraobserver correlation coefficients were 0.909 (95\% confidence interval, 0.8300.952 ) and 0.991 (95\% confidence interval, 0.983-0.996), and the interobserver coefficient was 0.965 (95\% confidence interval, 0.934-0.982).

\section{Statistical Analysis}

Data were analyzed using SPSS (version 20.0, IBM Corp, New York, NY). We used Pearson's correlation coefficient to analyze thoracic factors (sternal tilt angle and Haller index) and Cobb angle. The threshold for significance was a $p$ value of $<0.05$.

\section{Results}

Patients' mean age at presentation to our hospital was 10.3 years old (1-19 years old). There were 41 patients with idiopathic scoliosis, 29 patients with syndromic scoliosis, including 17 Marfan syndrome, 6 marfanoid types, 1 PraderWilli syndrome, 1 Sotos syndrome, 1 Down syndrome, and 3 others. The main areas of spinal curvature were thoracic in 52 patients, thoracolumbar in 10 , and lumbar in 8 . The mean Cobb angle of the main curve was 45.0 degrees (11109 degrees) (Table 1). The sternum was displaced to the left side in $72 \%$ of patients, central in $23 \%$, and to the right in $5 \%$. As for the sternal inclination, 23 patients had a right

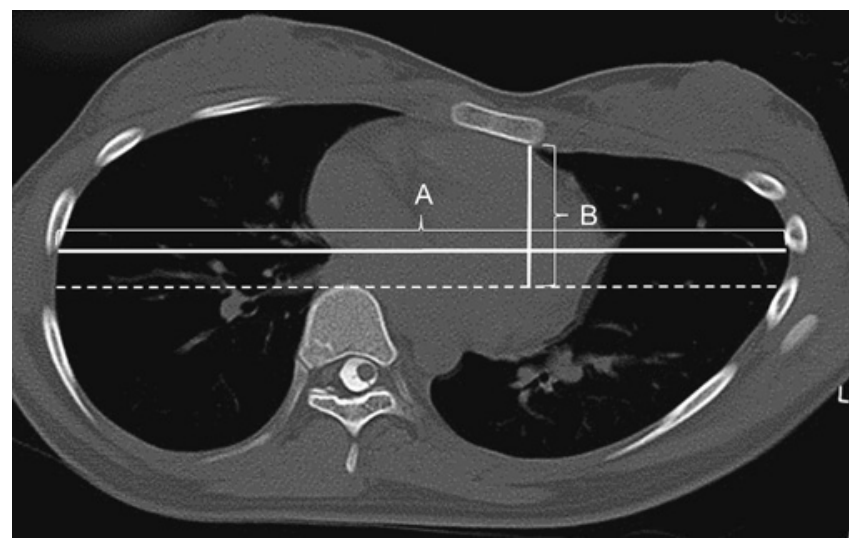

Figure 2. Modified Haller index. Axial CT scan showing positions for measuring. $\mathrm{A}=$ distance of the maximal inner transverse thoracic diameter. $\mathrm{B}=$ distance between the parallel line on the vertebra to the deepest point of the sternum at the most depressed plane of the sternum. 
Table 1. Patient's Characteristics.

\begin{tabular}{lc}
\hline Case & $\mathrm{n}=70$ \\
Age $\left(1^{\text {st }}\right.$ visit $)$ & $10.3(1 \sim 19)$ \\
Sex (male/female) & $37 / 33$ \\
Types of scoliosis & \\
Idiopathic & 41 \\
syndromic & 29 \\
Main curve & 52 \\
thoracic & 10 \\
thoracolumbar & 8 \\
lumbar & Ave. $45.0^{\circ}\left(11 \sim 109^{\circ}\right)$ \\
Cobb angle &
\end{tabular}

Table 2. Thoracic Parameters in Patients with Both Scoliosis and Pectus Excavatum.

\begin{tabular}{ll}
\hline $\begin{array}{l}\text { Thoracic parameters } \\
\text { Cases }\end{array}$ & $\mathrm{n}=43$ \\
Location of sternum & left 31 \\
& central 10 \\
& right 2 \\
& right 21 \\
left 22 \\
Sternal inclination & Ave. $12.4^{\circ}\left(1.8 \sim 34^{\circ}\right)^{*}$ \\
Avernal tilt angle $4.9(2.9 \sim 9.2)^{*}$ \\
Haller index & Ave \\
*29 cases which could be evaluated about all parameters \\
\multicolumn{2}{l}{ by CT scan of the thorax. }
\end{tabular}

inclination, and 22 had a left inclination among 45 patients who could be evaluated by CT scan. The mean sternal tilt angle was 12.4 degrees (1.8-34 degrees) and mean Haller index was 4.9 (2.9-9.2) among 29 patients who had their parameters evaluated by a CT scan of the whole chest area. (Table 2).

There was no significant correlation between Cobb angle and thoracic factors (sternal tilt angle and Haller index); pvalues were 0.371 and 0.159 , respectively. However, with regard to the severity of the pectus excavatum, a significant difference was found between sternal tilt angle and Haller index $(\mathrm{p}=0.009)$ (Table 3$)$. Twenty-four patients $(34 \%)$ received surgical correction of their pectus excavatum. In the scoliosis surgery group (27 patients), 16 patients had surgical correction of their pectus excavatum prior to scoliosis surgery, and 2 of the 14 patients in the scoliosis cast/brace treatment group had pectus excavatum surgery prior to applying the cast or brace (Fig. 3). The Nuss procedure using a chest bar or open repair procedure such as Ravitch procedure were performed for the pectus excavatum. Regardless of whether pectus excavatum surgery was done before scoliosis treatment, none of the patients experienced severe hypotension or cardiopulmonary dysfunction during scoliosis correction surgery or cast or brace treatment.
Table 3. Correlation between Cobb Angle and Thoracic Parameters.

\begin{tabular}{llccc}
\hline & & $\begin{array}{c}\text { Cobb } \\
\text { angle }\end{array}$ & $\begin{array}{c}\text { Sternal tilt } \\
\text { angle }\end{array}$ & $\begin{array}{c}\text { Haller } \\
\text { index }\end{array}$ \\
\hline Cobb angle & r & - & 0.172 & 0.269 \\
Sternal tilt angle & P value & - & 0.371 & 0.159 \\
& P value & - & - & 0.473 \\
& & - & - & 0.009 \\
\hline
\end{tabular}

\section{Discussion}

This study presents the clinical features and thoracic factors, such as sternal tilt angle and Haller index, seen in 70 patients diagnosed with scoliosis associated with pectus excavatum. Most of these patients had a left side deviated sternum and a higher Haller index score. Although the Cobb angle and thoracic parameters did not significantly correlate with each other, with regard to the severity of the pectus excavatum, a significant difference was found between sternal tilt angle and Haller index. Regardless of whether pectus excavatum surgery was performed before scoliosis treatment, none of our patients experienced severe hypotension or cardiopulmonary dysfunction during their scoliosis correction surgery or cast or brace treatment.

Several studies have reported the incidence of scoliosis in association with pectus excavatum. In 1989, Waters P et al. evaluated the incidence of scoliosis in 461 patients with pectus excavatum. They found that 99 of their study's patients (21.5\%) had scoliosis with an average Cobb angle of 15 degrees (range 6-78 degrees) ${ }^{9}$. Hong JY et al. examined 248 patients diagnosed with pectus excavatum for the incidence of scoliosis, and the prevalence of idiopathic scoliosis was $22.58 \%$. Furthermore, the incidence of scoliosis was significantly higher in female patients, and the Lenke type 1 predominated in pectus patients $(48.2 \%)^{6}$. Similarly, Wang Y et al. reported that 25 out of 142 patients $(17.6 \%)$ with pectus excavatum had scoliosis with a Cobb angle $>10$ degrees, and in $80 \%$ of the cases the spinal column was bent to the right. They found that adolescents had a higher incidence than pediatric patients, and those with a Cobb angle greater than 10 degrees were mainly within the severe scoliosis group and had Haller indices $\geq 3.5^{8)}$.

On the other hand, several authors reported severe complications associated with the prone position during surgery in children with scoliosis and pectus excavatum. Alexianu D et al. reported the case of a child with neurofibromatosis, pectus excavatum, and rapidly progressive scoliosis who developed severe hypotension after placement in the prone position during elective posterior spinal fusion. Transesophageal echocardiogram (TEE) revealed compression of the right ventricle by the sternum due to the compression of the transverse bolsters. After repositioning the longitudinal bolsters, the patient remained hemodynamically stable with no compression of the right ventricle evident on the TEE, and the posterior fusion proceeded uneventfully ${ }^{19}$. Bafus BT 

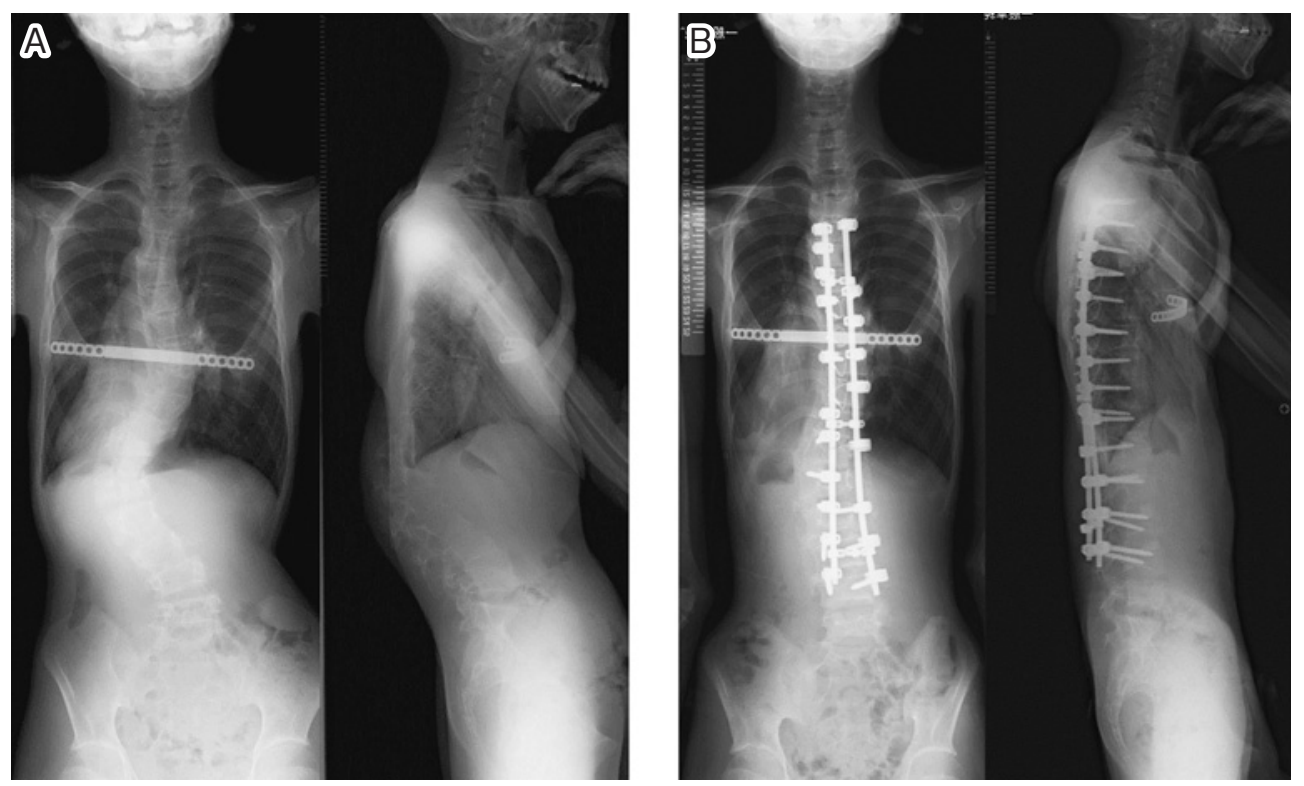

Figure 3. A 13-year-old male patient with syndromic scoliosis (Marfan syndrome) and pectus excavatum. He underwent anterior and posterior fusion surgery (T4/L4) after corrective surgery of the pectus excavatum.

A. Preoperative: thoracic 49 degrees, main thoracolumbar 64 degrees of coronal curvature in A-P and lateral radiographs.

B. Postoperative: thoracic 25 degrees, main thoracolumbar 21 degrees of coronal curvature in A-P and lateral radiographs.

et al. also reported a case of severe hypotension in a child with pectus excavatum who seemed marfanoid undergoing posterior spinal fusion for scoliosis after the patient was placed in the prone position. They accomplished the posterior spinal fusion for scoliosis after performing a modified Ravitch procedure to repair the pectus excavatum ${ }^{20)}$. Similarly, Galas JM et al. also presented a case with pectus excavatum and thoracolumbar scoliosis that developed severe hypotension after placement in the prone position for spinal fusion. A TEE showed the sternum compressing the right heart. After Nuss bar placement to correct the pectus excavatum, the patient underwent posterior spinal fusion uneventfully $^{21)}$.

Malek MH et al. performed a meta-analysis on cardiovascular function following surgical repair of pectus excavatum, and they demonstrated that surgical repair of the pectus excavatum significantly improves cardiovascular function ${ }^{22}$.

There were several limitations in our study. First, this was a retrospective study with a small sample size. We could not collect appropriate numbers of patients with each type of scoliosis. Second, we could not evaluate thoracic parameters in all the cases because some CT scans did not reveal the entire chest wall in the image. On the other hand, to the best of our knowledge, this is the first study to report thoracic factors in patients with both scoliosis and pectus excavatum which included idiopathic or syndromic type and varying degrees of scoliosis from moderate to severe which required treatment such as surgery or cast/brace. Finally, we suggest that the orthopedic surgeons fully assess the thoracic factors in patients with scoliosis and pectus excavatum prior to performing scoliosis surgery or cast/brace treatment and carefully monitor their patient's general condition during scoliosis treatment.

\section{Conclusion}

We evaluated 70 idiopathic or syndromic scoliosis patients who also had pectus excavatum. In most cases, the sternum was located on the left side, and their Haller index scores were higher than the normal population. Prone positioning, the corrective force applied during scoliosis surgery, and the thoracic cage compression that occurs during cast or brace treatment might negatively affect cardiac function in patients with both scoliosis and pectus excavatum.

Conflicts of Interest: The authors declare that there are no conflicts of interest.

Acknowledgment: We sincerely express our appreciation to Ms. S Hiraiwa and Michael Anton Castro for their contribution to this project.

\section{References}

1. Prats MR, Gonzalez LR, Venturelli MF, et al. Minimally invasive correction of pectus excavatum among adults. Report of eighteen cases. Rev Med Chil. 2009; 137(12): 1583-90.

2. Chang PY, Lai JY, Chen JC, et al. Long-term changes in bone and cartilage after Ravitch's thoracoplasty: findings from multislice computed tomography with 3-dimensional reconstruction. J Pediatr Surg. 2006; 41(12): 1947-50. 
3. Fokin AA, Steuerwald NM, Ahrens WA, et al. Anatomical, histologic, and genetic characteristics of congenital chest wall deformities. Semin Thorac Cardiovasc Surg. 2009; 21(1): 44-57.

4. Kotzot D, Schwabegger AH. Etiology of chest wall deformities--a genetic review for the treating physician. J Pediatr Surg. 2009; 44 (10): 2004-11.

5. Akcali Y, Ceyran H, Hasdiraz L. Chest wall deformities. Acta Chir Hung. 1999; 38(1): 1-3

6. Hong JY, Suh SW, Park HJ, et al. Correlations of adolescent idiopathic scoliosis and pectus excavatum. J Pediatr Orthop. 2011; 31 (8): 870-4.

7. Niedbala A, Adams M, Boswell WC, et al. Acquired thoracic scoliosis following minimally invasive repair of pectus excavatum. Am Surg. 2003; 69(6): 530-3.

8. Wang Y, Chen G, Xie L, et al. Mechanical factors play an important role in pectus excavatum with thoracic scoliosis. J Cardiothorac Surg. 2012; 7: 118.

9. Waters P, Welch K, Micheli LJ, et al. Scoliosis in children with pectus excavatum and pectus carinatum. J Pediatr Orthop. 1989; 9 (5): 551-6.

10. Gurnett CA, Alaee F, Bowcock A, et al. Genetic linkage localizes an adolescent idiopathic scoliosis and pectus excavatum gene to chromosome 18 q. Spine. 2009; 34(2): E94-100.

11. Golladay ES, Char F, Mollitt DL. Children with Marfan's syndrome and pectus excavatum. South Med J. 1985; 78(11): 131923.

12. Ho NC, Tran JR, Bektas A. Marfan's syndrome. Lancet. 2005; 366: $1978-81$

13. Le Parc JM, Molcard S, Tubach F, et al. Marfan syndrome and fibrillin disorders. Revue du Rhumatisme. 2000; 67(5): 401-7.

14. Redlinger RE Jr, Rushing GD, Moskowitz AD, et al. Minimally invasive repair of pectus excavatum in patients with Marfan syn- drome and marfanoid features. J Pediatr Surg. 2010; 45(1): 193-9.

15. Scherer LR, Arn PH, Dressel DA, et al. Surgical management of children and young adults with Marfan syndrome and pectus excavatum. J Pediatr Surg. 1988; 23(12): 1169-72.

16. Jaroszewski D, Notrica D, McMahon L, et al. Current management of pectus excavatum: a review and update of therapy and treatment recommendations. J Am Board Fam Med: JABFM. 2010; 23(2): 230-9.

17. Kelly RE Jr, Lawson ML, Paidas CN, et al. Pectus excavatum in a 112-year autopsy series: anatomic findings and the effect on survival. J Pediatr Surg. 2005; 40(8): 1275-8.

18. Blanco FC, Elliott ST, Sandler AD. Management of congenital chest wall deformities. Semin Plast Surg. 2011; 25(1): 107-16.

19. Alexianu D, Skolnick ET, Pinto AC, et al. Severe hypotension in the prone position in a child with neurofibromatosis, scoliosis and pectus excavatum presenting for posterior spinal fusion. Anesth Analg. 2004; 98(2): 334-5.

20. Bafus BT, Chiravuri D, van der Velde ME, et al. Severe hypotension associated with the prone position in a child with scoliosis and pectus excavatum undergoing posterior spinal fusion. J Spinal Disord Tech. 2008; 21(6): 451-4.

21. Galas JM, van der Velde ME, Chiravuri SD, et al. Echocardiographic diagnosis of right ventricular inflow compression associated with pectus excavatum during spinal fusion in prone position. Congenit Heart Dis. 2009; 4(3): 193-5.

22. Malek MH, Berger DE, Housh TJ, et al. Cardiovascular function following surgical repair of pectus excavatum: a metaanalysis. Chest. 2006; 130(2): 506-16.

Spine Surgery and Related Research is an Open Access article distributed under the Creative Commons Attribution - NonCommercial - NoDerivatives 4.0 International License. To view the details of this license, please visit (https://creativecommons.org/licenses/by - nc - nd/4.0/). 